PAPERS

\title{
A single-layer metallo-dielectric superstructure for enhancing the performances of EBG cavity antenna
}

\author{
Abdelhalim Chaabane ${ }^{1 *}$, Hussein Attia ${ }^{2}$, \\ Farid Djahli ${ }^{3}$, Tayeb A. Denidni ${ }^{4}$
}

\begin{abstract}
A novel single-layer metallo-dielectric superstructure is proposed in this paper. It is constructed by two asymmetric unitcells optimally arranged on the same layer to construct a partially reflective surface to be placed over a multilayer microstrip slot antenna named feed antenna for enhancing its performances. The radiation is expected to be maximum at the center of the formed layer. Thus, to maintain a high-gain performance, the unit-cells placed at the center are designed to provide a quasi-optimal reflection phase with high reflectivity at the frequency band of interest. A prototype of the proposed antenna operating at $10 \mathrm{GHz}$ with overall size of $2.133 \lambda_{0} \times 2.133 \lambda_{0} \times 0.56 \lambda_{0}$ is successfully designed and fabricated. The calculated and measured antenna gain results indicate that the proposed antenna exhibits a wider radiation bandwidth performance of about $41.15 \%$ and $36.15 \%$, respectively.
\end{abstract}

K e y w o r d s: wideband and high gain performances, wide radiation bandwidth, single layer partially reflective surface (PRS), electromagnetic-band-gap cavity antenna (ECA), equivalent circuit

\section{Introduction}

Microstrip antennas have many distinct and desirable attributes that make them the most known and broadly applied antennas in wireless communication systems. Unfortunately, some cramped parameters such as $3 \mathrm{~dB}$ gain and impedance bandwidths limit their applications $[1,2]$. Traditionally, one method to improve the antenna gain is using antenna arrays which may be constructed by many radiating elements. However, the feed network is usually complex and a lot of elements are needed to achieve a high gain. Furthermore, as the number of array elements increases, the overall size of the radiation system increases, the efficiency decreases, and an undesirable loss in the whole antenna structure occurs [3,4]. Electromagneticband-gap cavity antennas (ECA) also known as FabryPerot cavity antennas that were firstly suggested by Von Trentini in 1956, are well known for their capability to provide suitably directive radiation without need of a complicated feed system [5-7]. Usually, ECA is formed by a partially reflective surface (PRS) placed at a halfwavelength distance above a low gain primary source embedded in a perfect electric conductor. The gain improvement of the primary source is due to the cavity resonance and the multiple reflections of the waves between the PRS and the ground plane [8-10], the working principle of a conventional ECA is illustrated in Fig. 1. However, the performances of these antennas are highly sensitive to the cavity height formed by the ground plane and PRS.
An optimum gain at the broadside direction can be attained when the distance between the ground plane and the PRS is such that it causes the waves emanating from PRS to be in phase.

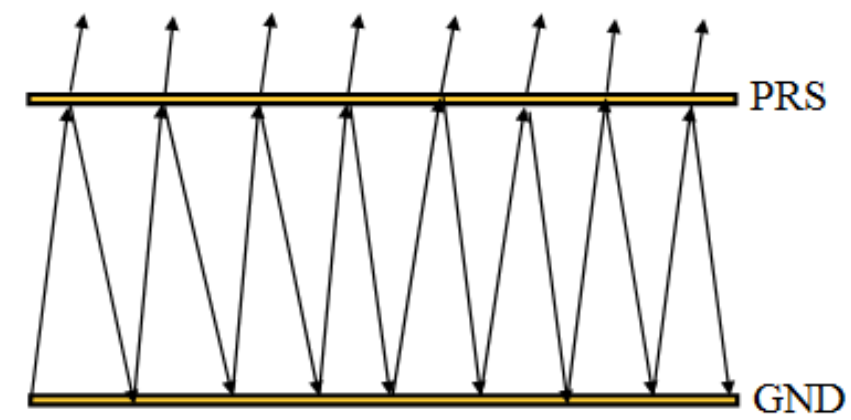

Fig. 1. Waves in the resonant cavity formed by a ground plane and PRS

For a given PRS, the resonance condition for the maximum broadside radiation can be formulated and calculated as given in [11]

$$
\varphi_{\mathrm{PRS}}+\pi(1-2 m)=2 k h
$$

where $\phi_{\mathrm{PRS}}$ is the reflection phase coefficient of the PRS, $k$ is the wave-number, and $m$ is an integer.

Accordingly, the cavity resonance condition over a wide frequency range yields a wide radiation bandwidth

\footnotetext{
${ }^{1}$ Laboratoire des Télécommunications-LT, Département d'Electronique et Télécommunications, Faculté des Sciences et de la Technologie, Université 8 Mai 1945 Guelma, BP 401, Guelma 24000, Algeria, ${ }^{2}$ King Fahd University of Petroleum and Minerals, Dhahran 31261, Saudi Arabia, ${ }^{3}$ Department of Electronics, LIS Laboratory, Faculty of Technology, University of Setif, 19000, Algeria, ${ }^{4}$ Institute National De La Recherche Scientifique (INRS) 800, Rue De La Guichetière Ouest, Montreal (Quebec), H5A1K6, Canada, * Corresponding author: abdelhalim.chaabane@univ-guelma.dz, chaabaneabdelhalim1979@gmail.com
} 


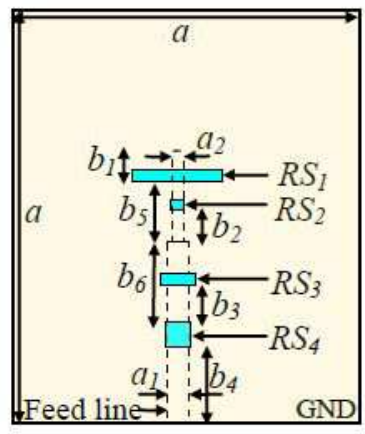

(a)

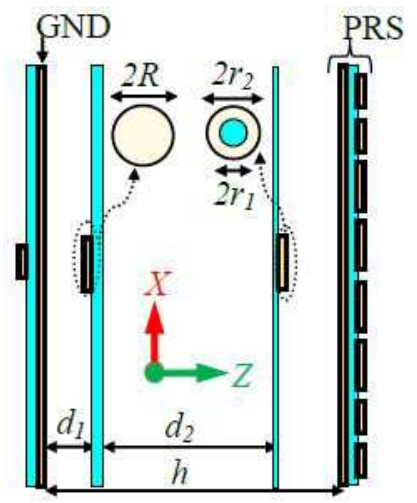

(b)
Fig. 2. Detailed configuration of the ECA (a) - top view of the ground plane of the feeding system (b) - cross section of the ECA

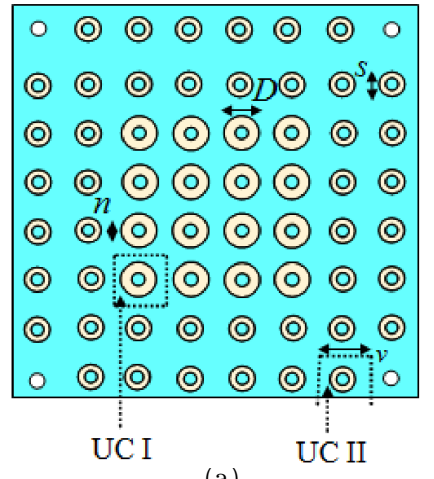

(a)

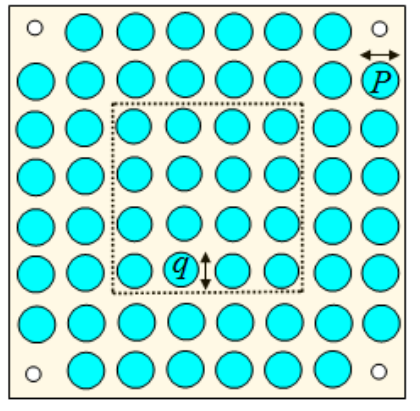

(b)
Fig. 3. Configuration of the proposed PRS-layer (a) - top view (b) - bottom view

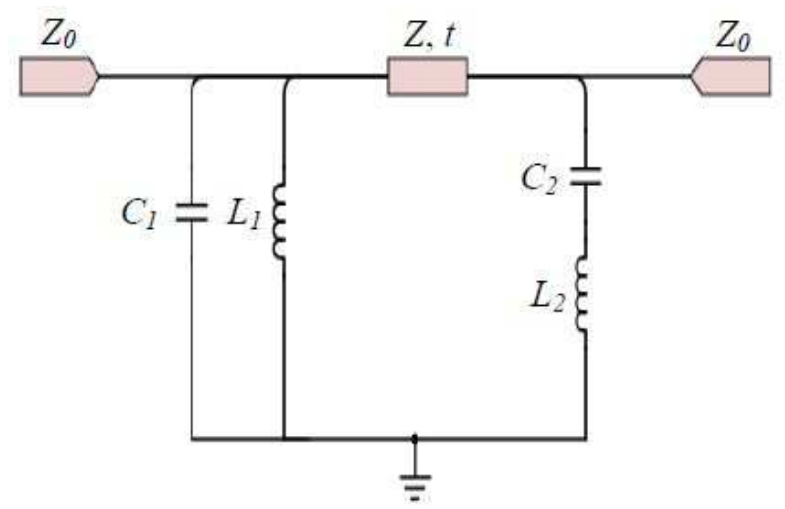

Fig. 4. Equivalent circuit for the proposed single layer unit-cells

performance for the ECA [12]. At the resonant frequency, the maximum boresight directivity is achieved and given by [13]

$$
D=\frac{1+R}{1-R}
$$

where $R$ is the magnitude of the reflection coefficient of the PRS. For a highly reflective surface, the directivity will be very high since the magnitude of the reflection coefficient of the PRS is close to 1.

Recently, many planar antennas based on different PRS structures have been proposed in literature for highly-directive radiation [14-20], but most of them exhibit poor $3 \mathrm{~dB}$ radiation bandwidth to be used for mod- ern telecommunication applications. In [14], two doublelayer PRS arrays of dissimilar dimensions are employed, each one consists of an AMC and a PRS surface printed on either side of a dielectric substrate; no more than $8 \%$ $3 \mathrm{~dB}$ radiation bandwidth has been achieved. In [15], two- and three-layers-PRS are placed over a dual-slot dual-resonant feeding structure; no more than $16 \% 3 \mathrm{~dB}$ radiation bandwidth has been achieved. In [16], three double-sided arrays are employed, each one consisting of an artificial impedance surface and a PRS surface printed on either sides of a dielectric substrate; a $3 \mathrm{~dB}$ gain bandwidth of $10.7 \%$ is achieved. In [17], three layers of PRSs are employed, consisting of metallic square patches and placed in front of a ground plane, forming three open cavities; a high gain performance is achieved by increasing the number of layers but no more than $15 \%$ radiation bandwidth is achieved. In [18], a double-layered printed unprinted dielectric superstrate was used as a PRS to enhance the gain bandwidth of a slot coupled patch antenna; no more than $25.8 \%$ radiation bandwidth has been achieved. In [19], a double-layer PRS with rectangular patch arrays with different sizes is employed over a broadband T-shaped phase shifter and a complex feeding network; the $3 \mathrm{~dB}$ gain bandwidth achieved is around $28.3 \%$. In [20], one PRS layer with uniform metallisation is used over a slot coupled-patch antenna; but only $28 \%$ $3 \mathrm{~dB}$ gain bandwidth is achieved.

In this paper, instead of using different metallisation on several layers, an array of unit-cells with dissimilar sizes is printed on the same layer to construct a single PRS-layer to place it as a cover of a feeding antenna, which is a planar microstrip slot-coupled antenna, constructed by three printed layers. The structure of the proposed ECA (feeding antenna with PRS) is presented in Fig. 2.

The designed PRS-layer is investigated as a superstructure to increase the performance of the feeding antenna. Wider calculated and measured radiation bandwidths of about $41.13 \%$ and $36.15 \%$, respectively, were achieved with the proposed ECA. Theoretical results were firstly obtained by the electromagnetic software Computer Simulation Technology (CST), Microwave Studio ${ }^{\text {TM }}[21]$.

\section{PRS-layer configuration and design approach}

Two different arrays formed by two different unit-cells are arranged on the same layer to construct only one PRSlayer. The detailed arrangement of unit-cells on the PRSlayer is shown in Fig. 3. An array of inductive square patches with dissimilar sizes, incorporated circular apertures, is printed on the bottom face of the PRS-layer, whereas another array of capacitive annular ring patches with dissimilar sizes is printed on the top face of the PRSlayer. The proposed single PRS-layer is designed on the Rogers RT/Duroid 6002 dielectric substrate of thickness $0.762 \mathrm{~mm}$, relative permittivity of 2.94 , and dielectric loss tangent of 0.0012 . 


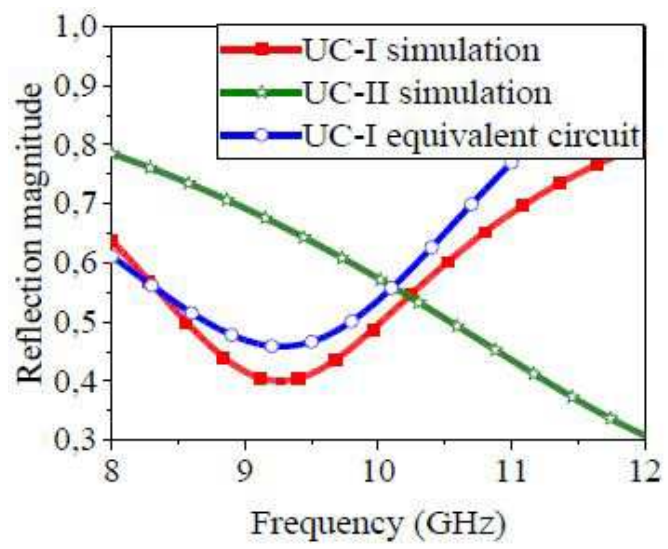

(a)

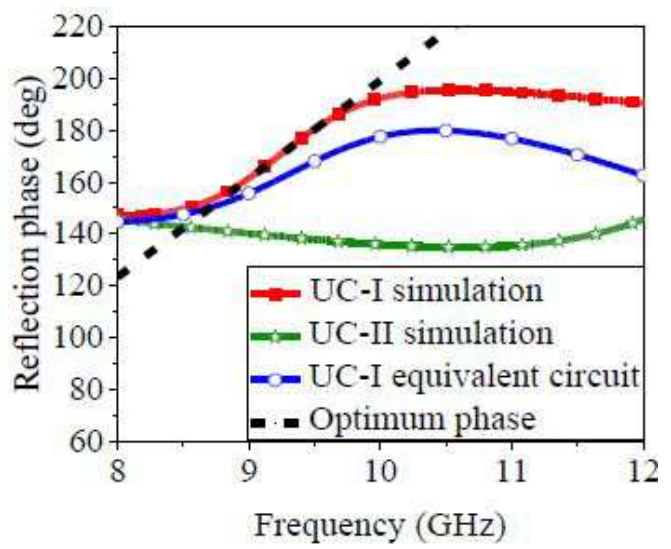

(b)

Fig. 5. Complex reflection coefficient for the two PRS unit cells (a) - reflection magnitude (b) - reflection phase

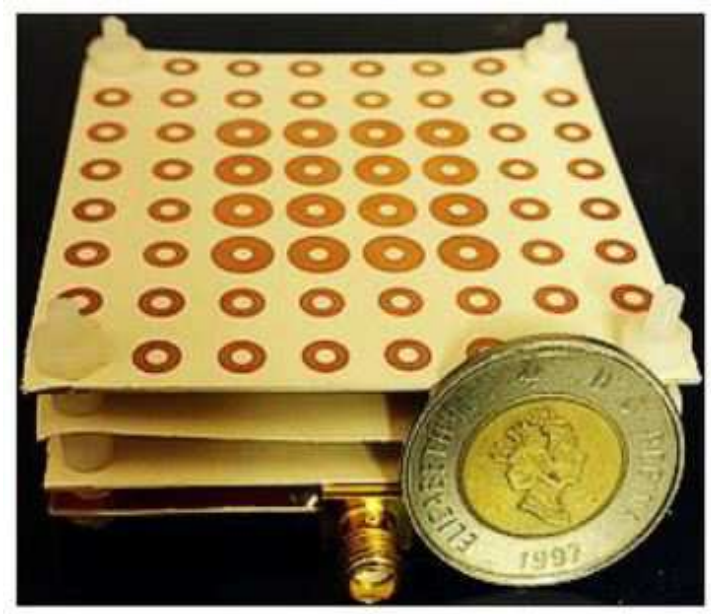

Fig. 6. Assembled prototype of the proposed ECA

The main goal of this work is a further improvement of the radiation bandwidth employing just a single PRSlayer. The design of the PRS unit-cells is the key for achieving such desired goal. The radiation is expected to be maximum at the center of the structure, thus, the antenna is influenced mainly by the performances of the $4 \times 4$ unit-cells UC-I placed at the centre. Therefore, an increasing reflection phase performance over a large frequency band in the operational frequency for UC-I is necessary to maintain a high-gain performance over a wide frequency range.

In the following, referring to the approaches introduced in [20] and [22], an equivalent circuit for the general structure of the unit-cells is established, as shown in Fig. 4. Moreover, the dielectric substrate constructing the unit-cells is modelled as a transmission line section that is characterized by its characteristic impedance $Z$ and length $t$. The characteristic impedance and length of this transmission line are assumed to be $Z=Z_{0} / \sqrt{\varepsilon_{r}}$ and $t=t_{0} \cdot \sqrt{\varepsilon_{r}}$, respectively, where $t_{0}$ is the substrate thickness, $\varepsilon_{r}$ is the relative permittivity of the dielectric, and $Z_{0}$ is free-space wave impedance. To validate the equivalent circuit model, the unit-cell UC-I placed in the centre of the PRS-layer is modelled. The estimated values of the electrical components, corresponding to the printed patches of the unit-cell, $L_{1,2}$ and $C_{1,2}$, were firstly calculated according to their physical dimensions by using the formulas given in [17] and [23]. Subsequently, the equivalent circuit was simulated and tuned with Agilent Advanced Design System (ADS) software [24].

The complex reflection coefficient corresponding to the equivalent circuit of the unit-cell UC-I follows well the calculated one obtained by CST software for the infinite structure unit-cell assumption, as illustrated in Fig. 5. All the calculated values of the electrical components in Fig. 4 of the unit-cell UC-I are set as follows: $L_{1}=$ $2.04 \mathrm{nH}, C_{1}=0.03 \mathrm{pF}, C_{2}=0.021 \mathrm{pF}, L_{2}=7.42 \mathrm{nH}$, $Z=219.87 \Omega, t=1.31 \mathrm{~mm}, Z_{0}=377 \Omega$.

An increasing PRS reflection phase response with frequency over a wide frequency band can yield a broad radiation gain bandwidth performance for the ECA. As well known, as the radiation bandwidth increases, gain reduction is inevitable. Accordingly, a compromised gain and radiation bandwidth performances is treated. The reflection phase of the unit-cell UC-I increases from $147.32^{\circ}$ to $195.53^{\circ}$ over a wide frequency range from 8.08 to $10.59 \mathrm{GHz}$, while the reflection phase of the unit-cell UCII is the continuity of the unit-cell UC-I, it starts increasing from $10.62 \mathrm{GHz}$ to over the interesting frequency bandwidth, as illustrated in Fig. 5.

\section{ECA design and measurement}

The designed unit-cells in infinite structures assumption are rearranged in one layer, as illustrated in Fig. 3, and placed over the feed antenna to achieve the desired performance (ie larger radiation bandwidth). Thus, an ECA is formed, which is constructed by four printed layers separated by air-gaps (3 layers of feeding antenna with 1 layer of PRS), as illustrated in Fig. 2. The four printed layers have a permittivity of 2.94 and thickness of $0.762 \mathrm{~mm}$, except the third layer, in which the parasitic ring patch is printed, which has the thickness of $0.127 \mathrm{~mm}$. A circular patch of radius $R$ is printed 


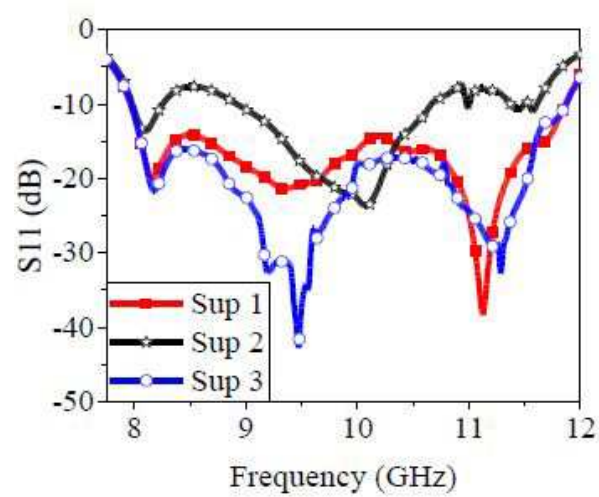

(a)

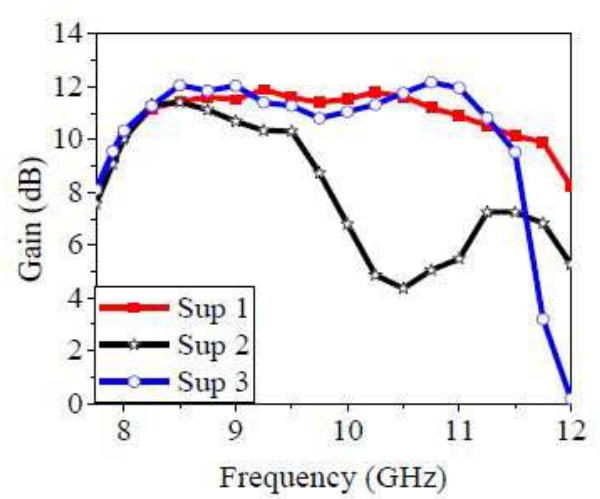

(b)

Fig. 7. Performances of the ECA for different configuration of metallisation, Sup 1: an array of UCI surrounded by an array of UC II (see Fig. 2), Sup 2: PRS-layer is constructed only by an array of UC II (uniform), Sup 3: PRS layer is constructed only by an array of UC I (uniform): (a) - reflection coefficient, (b) - gain

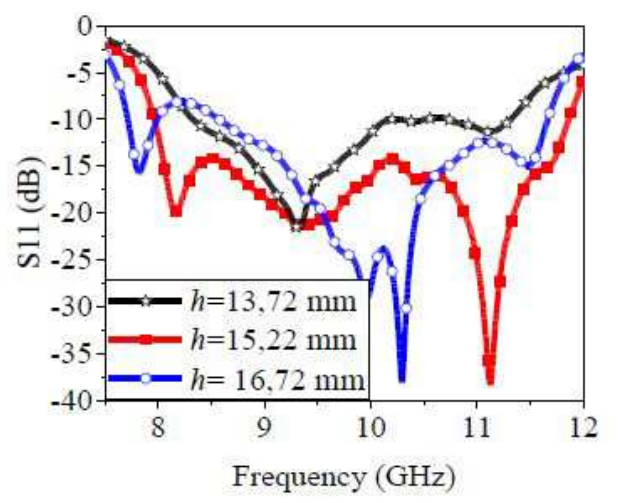

(a)

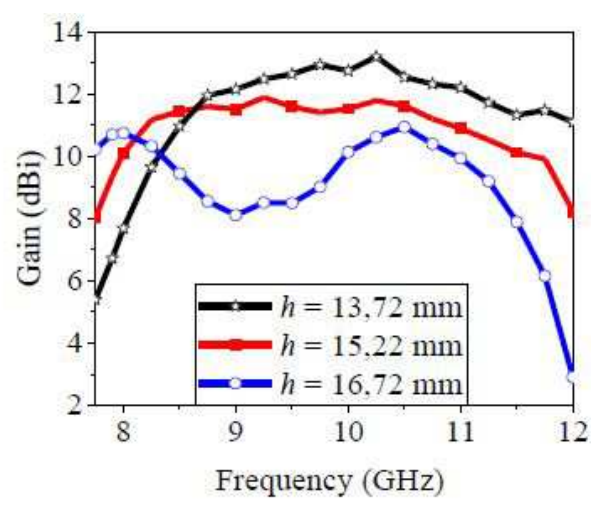

(b)

Fig. 8. Performance of the ECA for different thickness of the cavity between the PRS-layer and ground plane: (a) - reflection coefficient, (b) - gain

on the second layer of the feeding antenna, fixed at the distance of $d_{1}$ from the ground plane, and coupled to the feed-line. The feed line is printed on the backside of the lower layer and coupled with the circular patch through a rectangular slit $R S_{1}$ that is incorporated in the ground plane. Additionally, to enhance the impedance bandwidth, three other slits $R S_{2}, R S_{3}$ and $R S_{4}$ are inserted in the ground plane, as depicted in Fig. 2(a). Moreover, to improve the matching, an annular ring parasitic patch with inner radius of $r_{1}$ and outer radius of $r_{2}$ is fixed in the front of the feeding antenna, as depicted in Fig. 2(b). The overall size of the proposed ECA is $64 \mathrm{~mm} \times 64 \mathrm{~mm} \times 16.8 \mathrm{~mm}$ or about $2.133 \lambda_{0} \times 2.133 \lambda_{0} \times 0.56 \lambda_{0}$, where $\lambda_{0}$ is the free-space wavelength at $10 \mathrm{GHz}$. All the geometrical dimensions of the proposed ECA are set as follows: $a=64 \mathrm{~mm}, b_{1}=$ $2.45 \mathrm{~mm}, b_{2}=1.55 \mathrm{~mm}, b_{3}=4.5 \mathrm{~mm}, b_{4}=15.1 \mathrm{~mm}$, $b_{5}=5.2 \mathrm{~mm}, b_{6}=9.5 \mathrm{~mm}, a_{1}=1.93 \mathrm{~mm}, a_{2}=1.3 \mathrm{~mm}$, $R S_{1}=8.4 \mathrm{~mm} \times 1.2 \mathrm{~mm}, R S_{2}=1 \mathrm{~mm} \times 0.3 \mathrm{~mm}, R S_{3}=$ $2.9 \mathrm{~mm} \times 1.1 \mathrm{~mm}, R S_{4}=2 \mathrm{~mm} \times 1.9 \mathrm{~mm}, h=15.22 \mathrm{~mm}$, $d_{1}=2.58 \mathrm{~mm}, d_{2}=7.25 \mathrm{~mm}, R=5.6 \mathrm{~mm}, r_{1}=3 \mathrm{~mm}$, $r_{2}=4 \mathrm{~mm}, D=6.66 \mathrm{~mm}, s=4.6 \mathrm{~mm}, n=2 \mathrm{~mm}$, $v=8 \mathrm{~mm}, P=7.5 \mathrm{~mm}, q=7.1 \mathrm{~mm}$.

Using the optimized dimensions cited above, the printed layers constructing the ECA were fabricated and connected to each other by nylon screws and spacers with dielectric permittivity of 3.4 and radius $1.46 \mathrm{~mm}$, the assembled prototype is illustrated in Fig. 6 .

The design of the two types of the unit-cells, in the last section, can help to predict the appropriate arrangement of these unit-cells on the PRS-layer to achieve the purposed set by this work. The radiation performance of the ECA depends mainly on the reflection coefficient of the unit-cells and radiation characteristic of feeding antenna. Because, the PRS-layer is constructed by dissimilar size unit-cells. Thus, their arrangement on the PRS-layer have great influence on the performance of the antenna. The effect of the apportionment of the unit-cells UC-I and UC-II in the PRS-layer on the performance of the feed antenna is discussed. Compared to the configurations of the PRS-layer without combination of the two types of unit-cells, the arrangement of a total of $4 \times 4$ UC-I unitcells at the centre of the structure, with periodicity $v$, and surrounded by the unit-cell UC-II along the PRS-layer allowed to enhance the performance of the feed antenna, as illustrated in Fig. 7. An enhancement of upper than $3 \%$ radiation bandwidth is achieved with the use of Sup 1.

Figure 8 shows the results of the parameter study of the thickness $h$ of the cavity formed by the ground plane and the PRS-layer on the performances of the antenna (impedance matching and gain). With increasing $h$ from $13.72 \mathrm{~mm}$ to $16.72 \mathrm{~mm}$, the impedance matching of the 


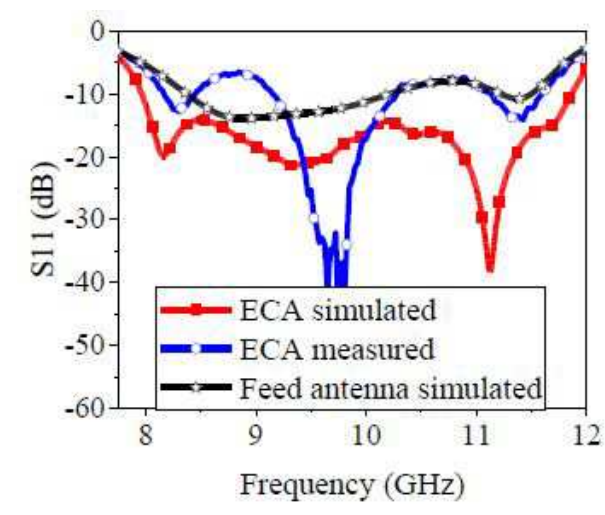

(a)

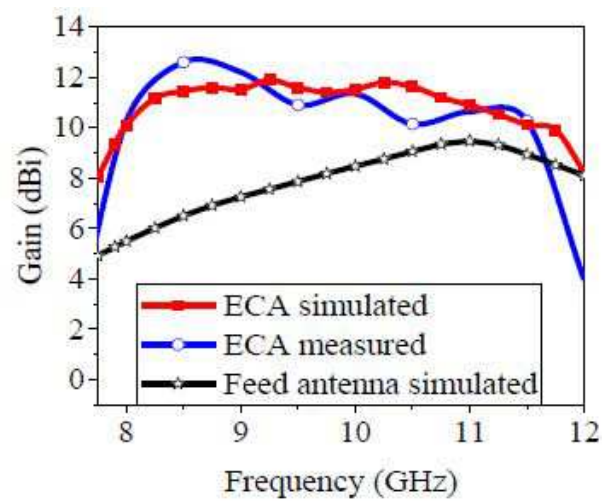

(b)

Fig. 9. Performance of the ECA versus the feeding antenna (FA): (a) - reflection coefficient, (b) - gain

antenna becomes better, and improvements of the gain and radiation bandwidth are obtained. Because the goal of this work is to achieve a larger radiation bandwidth with fairly high-gain in the operating frequency, the value of the $15.22 \mathrm{~mm}$ is chosen as the optimum high. By placing the proposed PRS-layer at the optimum thickness, further improvements of the impedance matching, radiation bandwidths, and antenna gain of the feed antenna are achieved.

The influence of the PRS-layer on the performances of the feeding antenna is illustrated in Fig. 9. Fig. 9(a) shows that the PRS has a great effect on the impedance bandwidth of the feeding antenna, where a considerable improvement is observed. Furthermore, the simulated input matching bandwidth of the ECA covers the frequency band from 7.96 to $11.87 \mathrm{GHz}$, while the measured one obtained by an Agilent 8722 ES vector network analyzer shows that the fabricated prototype covers the frequency band from around 8.02 to $11.78 \mathrm{GHz}$ for the limit of $6.54 \mathrm{~dB}$. The measured gain at far-field of the ECA was carried out in a full anechoic chamber, showing a good agreement with the simulated one, as illustrated in Fig. 9(b). Additionally, covering the feed antenna by the PRS-layer, a great enhancement on its radiation performance is obtained; the gain response is greatly improved and a larger $3 \mathrm{~dB}$ gain bandwidth is achieved. A calculated $41.13 \% 3 \mathrm{~dB}$ gain bandwidth is achieved, whereas the measured one is about $36.15 \%$ with a maximum gain of $12.6 \mathrm{dBi}$. Essentially, the ECA is purposely designed to obtain a larger radiation bandwidth; thus it is reasonably natural to do not have a higher gain performance. Slight mismatch between the simulated and measured results is attributed to the fabrication inaccuracies, antenna assembly, feed connector welding, or measurement tolerance.

The normalized radiation patterns of the prototype antenna were measured in an anechoic chamber for different operating frequencies $(8.5,9,10 \mathrm{GHz})$. A double ridged horn antenna (model AH-118 operating in $1-18 \mathrm{GHz}$ ) is used for transmitting electromagnetic waves, whereas the proposed ECA is used for receiving. The measured and simulated radiation patterns for both E- and H-planes are presented in Fig. 10. As can be seen, the measured radiation patterns are consistent with the designed ones. Both numerical and experimental side-lobe level (SLL) results are below than $-10 \mathrm{~dB}$ at almost the radiation bandwidth. Furthermore, an excellent cross polarization performance was measured, and it is almost below $-20 \mathrm{~dB}$ throughout the frequency band studied, which demonstrates the good antenna performance. The performances of the fabricated antenna are compared with some other recently published antenna in Tab. 1. As it can be seen, the proposed antenna provides the widest radiation bandwidth and the wider working bandwidth by the use of only one layer in superstructure. Thus, it could be concluded that the designed PRS superstructure can be appropriate for enhancing the performances of printed antennas operating in X-band.

Table 1. A comparative study between the proposed antenna and some recently published work operating in $\mathrm{X}$-band

\begin{tabular}{lcccc}
\hline Refs & $\begin{array}{c}\text { No. of } \\
\text { layers }\end{array}$ & $\begin{array}{c}3 \mathrm{~dB} \\
\text { gain BW }(\%)\end{array}$ & $\begin{array}{c}\text { Max. gain } \\
(\mathrm{dB})\end{array}$ & $\begin{array}{c}\mathrm{S}_{11} \\
\mathrm{BW}(\%)\end{array}$ \\
\hline$(19)$ & 2 & 28.3 & 14.7 & 33.16 \\
$(20)$ & 2 & 28 & 13.8 & 26.3 \\
$(25)$ & 2 & 33.33 & 13.57 & 35.53 \\
$(26)$ & 2 & 33.87 & 13.7 & 37.38 \\
This work & 1 & 39.43 & 12.6 & 37.98 \\
\hline
\end{tabular}

\section{Conclusion}

A novel single-layer metallo-dielectric superstructure has been presented in this paper for enhancing the performances of a feeding antenna operating in X-band. The advantages of the constructed ECA are threefold: 1) a wider radiation bandwidth with a reasonable high gain performance, 2) a consistent radiation pattern over almost the radiation bandwidth, and 3) two PRS unit-cells printed in one layer to reduce the size and the cost. Wider radiation bandwidth of about $41.13 \%$ and $39.43 \%$ have 


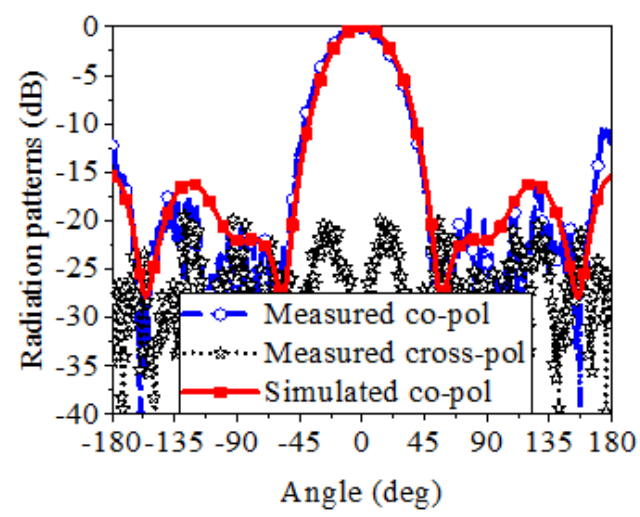

(a)

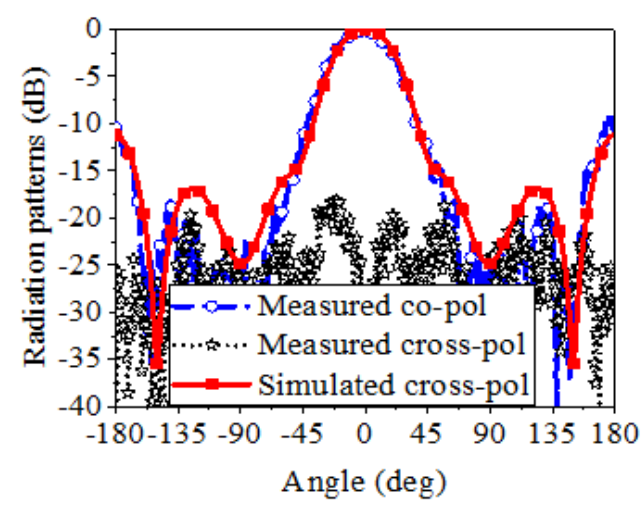

(c)

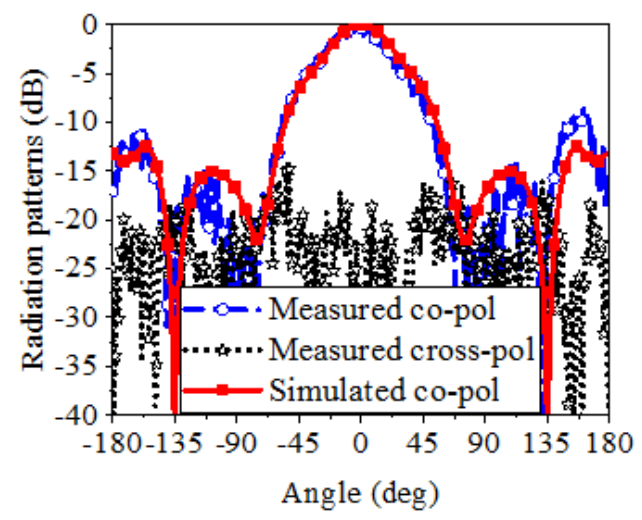

(e)

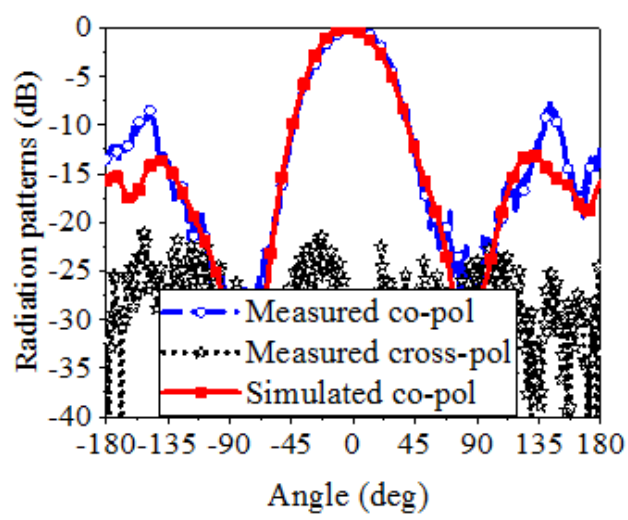

(b)

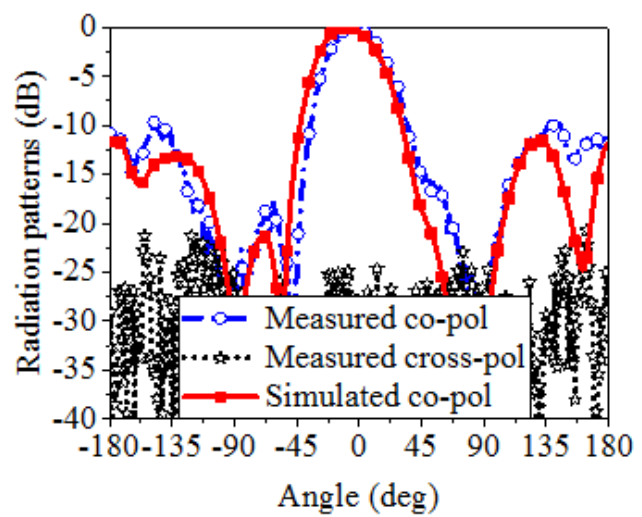

(d)

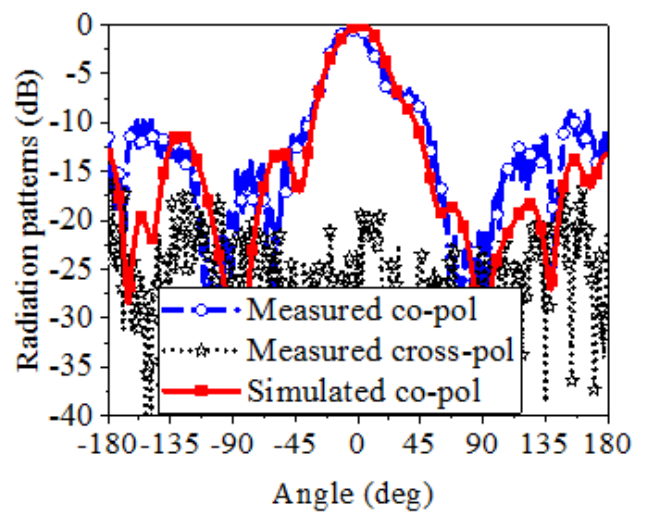

(f)

Fig. 10. Measured and simulated radiation patterns in both $\mathrm{H}$ - and E-planes at three operating frequencies of the ECA: (a) - H- plane at 8.5 GHz, (b) - E-plane at $8.5 \mathrm{GHz},(\mathrm{c})-\mathrm{H}$ - plane at $9 \mathrm{GHz},(\mathrm{d})-$ E-plane at $9 \mathrm{GHz},(\mathrm{e})$ - H-plane at $10 \mathrm{GHz},(\mathrm{f})-\mathrm{E}-\mathrm{plane}$ at $10 \mathrm{GHz}$

been calculated and measured, respectively. The calculated and measured impedance bandwidths are extending from 7.96 to $11.87 \mathrm{GHz}$ and from 8.02 to $11.78 \mathrm{GHz}$, respectively. Compared to the feed antenna, these performances are greatly enlarged. The proposed concept has proven its ability to exhibit a larger radiation bandwidth, which makes it a good candidate for many wireless applications.

\section{Acknowledgment}

This work was supported by the Directorate-General for Scientific Research and Technological Development (DG-RSDT) of Algeria.

\section{REFERENCES}

[1] D. Gangwar, S. Das, R. L. Yadava, and B. K. Kanaujia, "Circularly Polarized Inverted Stacked High Gain Antenna with Frequency Selective Surface", Microwave and Optical Technology Letters, vol. 58, no. 3, pp. 732-740, 2016.

[2] Y. Li, R. Mittra, B. Zeng, G. Lu, Z. Li, J. Liu, C. W. Chen, and D. C. Chang, "Directivity Enhancement of Fabry-Perot Antenna by Using a Stepped-Dielectric Slab Superstrate", Microwave and Optical Technology Letters, vol. 54, no. 3, pp. 711-715, 2012.

[3] B. P. Chacko, G. Augustin, and T. A. Denidni, "FPC Antennas, C-Band Point-to-Point Communication Systems", IEEE Antennas and Propagation Magazine, vol. 58, no. 1, pp. 56-64, 2016. 
[4] J. Ju and J. L. Choi, "Broadband High Gain Fabry-Perot Cavity Antenna with Back Radiation Reduction", Microwave and Optical Technology Letters, vol. 55, no. 5, pp. 975-978, 2013.

[5] R. M. Hashmi and K. P. Esselle, "A Wideband EBG Resonator Antenna with an Extremely Small Footprint Area", Microwave and Optical Technology Letters, vol. 57, no. 7, pp. 1531-1535, 2015.

[6] H. Boutayeb, K. Mahdjoubi, A. C. Tarot, and T. A. Denidni, "Directivity of an Antenna Embedded Inside a Fabry-Perot Cavity: Analysis and Design", Microwave and Optical Technology Letters, vol. 48, no. 1, pp. 12-17, 2006.

[7] B. A. Zeb and K. P. Esselle, "High-Gain Dual Band Dual-Polarised Electromagnetic Band Gap Resonator Antenna with All-Dielectric Superstrcture", IET Microwaves Antennas \& Propagation, vol. 9, no. 10, pp. 1059-1065, 2015.

[8] A. Chaabane, F. Djahli, H. Attia, and T. A. Denideni, "Radiation Bandwidth Improvement of Electromagnetic Band Gap Cavity Antenna", Frequenz vol. 71, no. 5-6, pp. 243-249, 2018.

[9] A. Pirhadi, M. Hakkak, F. Keshmiri, and R. K. Baee, "Design of Compact Dual Band High Directive Electromagnetic Bandgap (EBG) Resonator Antenna using Artificial Magnetic Conductor", IEEE Transactions on Antennas and Propagation, vol. 55, no. 6, pp. 1682-1690, 2007.

[10] H. Liu, S. Lei, X. Shi, and L. Li, "Study of Antenna Superstrates using Metamaterials for Directivity Enhancement Based on Fabry-Perot Resonant Cavity", International Journal of Antennas and Propagation, vol. 2013, Article ID 209741, pp. 1-10, 2013.

[11] A. R. Weily, T. S. Bird, and Y. J. Guo, "A Reconfigurable High-Gain Partially Reflecting Surface Antenna", IEEE Transactions on Antennas and Propagation, vol. 56, no. 11, pp. 3382-3390, 2008.

[12] M. L. Abdelghani, H. Attia, and T. A. Denidni, "Dual and Wide Band Fabry Pérot Resonator Antenna for WLAN Applications", IEEE Antennas and Wireless Propagation Letters, vol. 16, pp. 473-476, 2016.

[13] Z. G. Liu, W. X. Zhang, D. L. Fu, Y. Y. Gu, and Z. Ge, "Broadband Fabry-Perot Resonator Printed Antennas using FSS Superstrate with Dissimilar Size", Microwave and Optical Technology Letters, vol. 50, no. 6, pp. 1623-1627, 2008.

[14] K. Konstantinidis, A. P. Fresidis, and P. S. Hall, "Dual Subwavelength Fabry-Perot Cavities for Broadband Highly Directive Antennas", IEEE Antennas and Wireless Propagation Letters, vol. 13, pp. 1184-1186, 2014.

[15] K. Konstantinidis, A. P. Fresidis, and P. S. Hall, "Dual-Slot Feeding Technique for Broadband Fabry Perot Cavity Antennas", IET Microwaves Antennas \& Propagation, vol. 9, no. 9, pp. 861-866, 2015.

[16] K. Konstantinidis, A. P. Fresidis, and P. S. Hall, "Broadband Sub-Wavelength Profile High Gain Antennas Based on Multi-Layer Metasurfaces", IEEE Transactions on Antennas and Propagation, vol. 63, no. 1, pp. 423-427, 2015.

[17] K. Konstantinidis, A. P. Fresidis, and P. S. Hall, "Multilayer Partially Reflective Surfaces for Broadband Fabry-Perot Cavity Antennas", IEEE Transactions on Antennas and Propagation, vol. 62, no. 7, pp. 3474-3481, 2014.

[18] N. Wang, J. Li, G. Wei, and L. Talbi, "Wideband Fabry-Perot Resonator Antenna with Two Layers of Dielectric Superstrates", IEEE Antennas and Wireless Propagation Letters, vol. 14, pp. 229-232, 2014.

[19] F. Qin, S. Gao, G. Wei, and J. Xu, "Broadband Circularly Polarized Fabry-Perot Antenna Integrated with Wideband Phase Shifter for Satellite Communication", Microwave and Optical Technology Letters, vol. 58, no. 5, pp. 1109-1113, 2016.

[20] N. Wang, Q. Liu, C. Wu, L. Talbi, Q. Zeng, and J. Xu, "Wideband Fabry-Perot Resonator Antenna with Two Complementary FSS Layers", IEEE Transactions on Antennas and Propagation, vol. 62, no. 5, pp. 2463-2471, 2014.
[21] CST Microwave Studio, "CST: Computer Simulation Technologies", version 2015.

$22]$ Z. L. Wang, K. Hashimoto, N. Shinohara, and H. Matsumoto, "Frequency-Selective Surface for Microwave Power Transmission", IEEE Transactions on Microwave Theory and Techniques, vol. 47, no. 10, pp. 2039-2041, 1999.

[23] A. Ebrahimi, S. Nirantar, W. Withayachumnankul, M. Bhaskaran, S. Sriram, S. F. Al Sarawi, and D. Abbott, "Second Order Terahertz Band Pass Frequency Selective Surface with Miniaturized Elements", IEEE Transactions on Terahertz Science and Technology vol. 5, no. 5, pp. 761-769, 2015.

24] ADS Software, "ADS: Advanced Design System, Electronic Design Automation Software", version 2015.01.

[25] A. Chaabane, F. Djahli, H. Attia, L. M. Abdelghani, and T. A. Denideni, "Wideband and High-Gain EBG Resonator Antenna Based on Dual Layer PRS", Microwave and Optical Technology Letters, vol. 59, no. 1, pp. 98-101, 2017.

26] A. Chaabane, F. Djahli, H. Attia, and T. A. Denideni, "Antenna Radiation Bandwidth Broadening using Wideband Double-Layer Partially Reflective Surfaces", IEEE $17^{\text {th }}$ International Symposium on Antenna Technology and Applied Electromagnetics (ANTEM, Montreal, Canada, 10-13 July, 2016.

Received 4 September 2020

Abdelhalim Chaabane received the doctorate degree in Electronics from the University of Setif-1 Algeria, in 2017. He is currently an Associate Professor with the Département d'Electronique \& Télćommunications, Université 8 Mai 1945 Guelma. He is a member of Telecommunications Laboratory, Université 8 Mai 1945 Guelma, Algeria. His main research interests includes Fractal antennas, Ultra-wideband antennas and Metamaterial antennas.

Hussein Attia received the $\mathrm{PhD}$ degree in electrical and computer engineering from the University of Waterloo, Ontario, Canada, in 2011. After finishing his $\mathrm{PhD}$, he worked as a Research Engineer with the Coding and Signal Transmission Laboratory, University of Waterloo, from March 2011 to July 2013. He was granted a Postdoctoral Fellowship at Concordia University, Montreal, Quebec, from August 2014 to July 2015. Also, he was a Visiting Scholar with the University de Quebec (INRS), in 2015 and 2017, respectively. He is currently an Assistant Professor with the King Fahd University of Petroleum and Minerals (KFUPM). He has published more than 60 journal and conference papers. His research interests include millimeter-wave high-gain and wide-band antennas, analytical techniques for electromagnetic modeling, and engineered magnetic metamaterials. Dr Attia, during his $\mathrm{PhD}$ program, received the University of Waterloo Graduate Scholarship for excellence in research and coursework, in 2009. He was a finalist in the Student Paper Competition of the 2011 IEEE AP-S International Symposium on Antennas and Propagation. He ranked first among all BSc. students of Electronics and Communication Engineering, Zagazig University, Egypt, in 1999.

Farid Djahli received the electronics engineering diploma in 1981 and the MS degree in electronics in 1985, from the ENPA (Ecole Nationale Polytechnique d'Alger). In 1992, he received the $\mathrm{PhD}$ in microelectronics from LPM of INSA, Lyon (Laboratoire de Physique de la Matire, Institut National des Sciences Appliquées de Lyon), France. He worked on the modelling of the degradation of MOSFETs using different techniques of characterization, especially the charge pumping techniques. He joined the Electronics Institute of the University of Setif, Algeria, in 1992, where he worked as a professor. 
In addition to microelectronics, his research interests include MMIC circuits, modelling of microstrip discontinuities, and microstrip antennas. He is the author and the co-author of more than 100 scientific papers.

Tayeb A. Denidni received M. Sc. and PhD degrees in electrical engineering from Laval University, Quebec City, QC, Canada, in 1990 and 1994, respectively. From 1994 to 2000, he was a Professor with the engineering department, Universit du Quebec in Rimouski (UQAR), Rimouski, QC, Canada, where he founded the Telecommunications laboratory. Since August 2000, he has been with the Institut National de la Recherche Scientifique (INRS), Universit du Quebec, Montreal, QC, Canada. He has established international recogni- tion in research and development in the area of antennas and zmicrowave and mm-wave circuit design. He has developed innovative efficient antenna designs and made significant contributions to their use in wireless communication systems at microwave and millimeter-wave bands. He has authored and co-authored of more than 700 technical peer reviewed papers in international journals and conference proceedings. His current research areas of interest include reconfigurable antennas using EBG and FSS structures, dielectric resonator antennas, metamaterial antennas, adaptive arrays, switched multi-beam antenna arrays, ultra-wideband antennas, microwave and development for wireless communications systems. 